\title{
Diagnostics of Non-thermal Particles in Solar Chromospheric Flares
}

\author{
C. Fang, Z. Xu, and M. D. Ding \\ Department of Astronomy, Nanjing University, Nanjing, 210093, China
}

\begin{abstract}
Particle beam bombardment on the solar chromosphere produces non-thermal ionization and excitation. The effect on hydrogen lines is investigated by using non-LTE theory and semi-empirical flare models. It has been found that in the case of electron bombardment, the $\mathrm{H} \alpha$ line is widely broadened and enhanced. Significant enhancements at the wings of $\operatorname{Ly} \alpha$ and $\operatorname{Ly} \beta$ lines are also predicted. In the case of proton bombardment, less strong broadening and less central reversal are expected. We found that the total energy flux of the particle beam and the atmospheric condition give much influence on the line profiles, which, however, are less sensitive to the power index. Based on the $\mathrm{H} \alpha$ line profile measurement, a method to deduce the total energy flux of the particle beam is proposed.
\end{abstract}

\section{Introduction}

Generally, during solar flares, the non-thermal electrons of more than $10 \mathrm{keV}$ can be detected by their X-ray and radio emission. While protons of more than $1 \mathrm{MeV}$ can be detected by their $\gamma$-ray line emission. However, it turns out that non-thermal electrons and protons of energy as low as $100 \mathrm{keV}$ can be diagnosed by use of their UV and optical spectra.

The non-thermal particles bombardment can cause at least three effects: (1) non-thermal ionization and excitation (e.g. Chambe \& Hénoux 1979; Aboudarham \& Hénoux 1987; Fang et al. 1993 (thereafter referred to as Paper I); Hénoux et al. 1993 (thereafter referred to as Paper II); Xharkova \& Kobylinskii 1993; Kasparova \& Heinzel 2002);(2) proton-hydrogen charge exchange (e.g. Canfield \& Chang 1985; Fang et al. 1995); (3) impact line polarization (e.g. Kazantsev \& Hénoux 1995; Xu et al. 2003).

In this paper, we give our recent study on the first effect. A description of non-thermal excitation and ionization by particles is given in Section 2 . The computed $\mathrm{H} \alpha$, Ly $\alpha$ and $\operatorname{Ly} \beta$ line profiles are also given. Based on our new result, a method to deduce the particle total energy flux is proposed in Section 3. A conclusion is given in the last Section.

\section{Non-thermal Excitation and Ionization by Particle Beams}

\subsection{Non-thermal Excitation and Ionization Rates}

The rate of energy deposit in an atmosphere by an electron or proton beam is given by (Emslie 1978; Chambe \& Hénoux 1979) 


$$
\frac{d E^{H}}{d t}=\frac{1}{2}(1-x) n_{H} \Lambda^{\prime} \frac{K \mathcal{F}_{1}}{E_{1}^{2}}\left(\frac{N}{N_{1}}\right)^{-\frac{\delta}{2}}(\delta-2) \int_{0}^{u_{1}} \frac{u^{\frac{\delta}{2}-1} d u}{(1-u)^{\frac{2+\bar{\beta}}{4+\bar{\beta}}}}
$$

Where $x$ is the ionization degree. The particle flux is supposed to be proportional to $E^{-\delta}$, with a low energy cut-off $E_{1} . \mathcal{F}_{1}$ is the total energy flux above $E_{1} . N_{1}$ is the deepest column depth reached by the particles of energy $E_{1}$. A given column density $N$ can only be reached by particles of energy greater than $E_{N}$. $E_{N}=\left[(2+\bar{\beta} / 2) \bar{\gamma} K N / \mu_{0}\right]^{1 / 2}$, where all parameters are defined in Emslie's paper. The parameter $u$ is defined as $u=\left(E_{N} / E\right)^{2}$, so that $u_{1}=1$ for $N>N_{1}$; $u_{1}=N / N_{1}$ for $N \leq N_{1}$.

According to the theory given in Papers I and II, we have

$$
\frac{d E^{H}}{d t}=n_{1}\left(\sum \chi_{1 j} C_{1 j}^{B}+\chi_{1 c} C_{1 c}^{B}\right)
$$

where $\chi_{1 j}$ and $\chi_{1 c}$ are the hydrogen excitation and ionization potentials, respectively. $n_{1}$ is the population at the ground level of hydrogen atoms. Thus, with a four-levels-plus-continuum atomic model of hydrogen, the non-thermal excitation and ionization rates of hydrogen by electron or proton beams, $C_{1 j}^{B}$ and $C_{1 c}^{B}$, can be obtained as

$$
\begin{aligned}
& C_{12}^{B} \simeq 2.9410^{10} \frac{1}{n_{1}} \frac{d E^{H}}{d t}, \quad C_{13}^{B} \simeq 5.3510^{9} \frac{1}{n_{1}} \frac{d E^{H}}{d t}, \\
& C_{14}^{B} \simeq 1.9110^{9} \frac{1}{n_{1}} \frac{d E^{H}}{d t}, \quad C_{1 c}^{B} \simeq 1.7310^{10} \frac{1}{n_{1}} \frac{d E^{H}}{d t} .
\end{aligned}
$$

\subsection{Line Profile Computations}

The statistical equilibrium equation, including the non-thermal excitation and ionization rates, and the transfer equation, coupled with the hydrostatic equilibrium and the particle conservation equations, can be solved iteratively.

In order to show the influence of the non-thermal effects on different atmospheres, we used three semi-empirical atmospheric models, i.e. the quiet-Sun VAL3C model given by Vernazza et al. (1981), and the two flare models of F1 and F2 given by Machado et al. (1980). The non-thermal $\mathrm{H} \alpha$, Ly $\alpha$, and $\mathrm{Ly} \beta$ line profiles were computed for various values of the total energy flux $\mathcal{F}_{1}$ and the power index $\delta$. Different from the Papers I and II, the partial frequency redistribution was included in the Ly $\alpha$ line and the iteration number was increased to more than 12000 to assure a high precision of about $110^{-4}$.

Figure 1 shows the new result of computed $\mathrm{H} \alpha$, Ly $\alpha$ and $\operatorname{Ly} \beta$ line profiles under an electron beam bombardment with $\delta=4$ and different values of $\mathcal{F}_{1}$. Figure 2 gives the line profiles for the different models bombarded by electron and proton beams. An energy flux $\mathcal{F}_{1}=510^{11}$ and $510^{10} \mathrm{erg} \mathrm{cm}^{-2} \mathrm{~s}^{-1}$ for the electron and proton beam, respectively, was adopted. In all figures, a Gaussian macroturbulence velocity of $20 \mathrm{~km} \mathrm{~s}^{-1}$ was adopted to convolve the non-thermal line profiles. It can be seen from these figures that the intensity enhancement at 

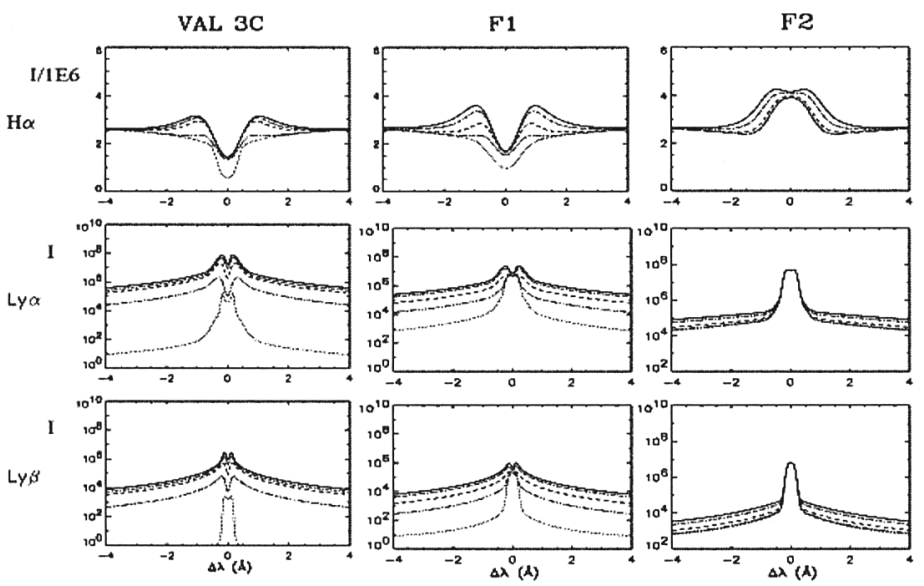

Figure 1. $\operatorname{H} \alpha, \operatorname{Ly} \alpha$ and $\operatorname{Ly} \beta$ line profiles for the three models and for $\delta=4$ of an electron beam bombardment with $\mathcal{F}_{1}=110^{12}$ (full line), 5 $10^{11}$ (dotted-dashed line), $110^{11}$ (dashed line) and $110^{10}$ (three dotted per dashed line) erg cm $\mathrm{cm}^{-2} \mathrm{~s}^{-1}$. The low energy cut-off $E_{1}$ is taken to be $20 \mathrm{keV}$. Dotted lines are for the same model but without including the non-thermal effects. The unit of $I$ is $\mathrm{erg} \mathrm{s}^{-1} \mathrm{~cm}^{-2} \mathrm{sr}^{-1} \AA^{-1}$
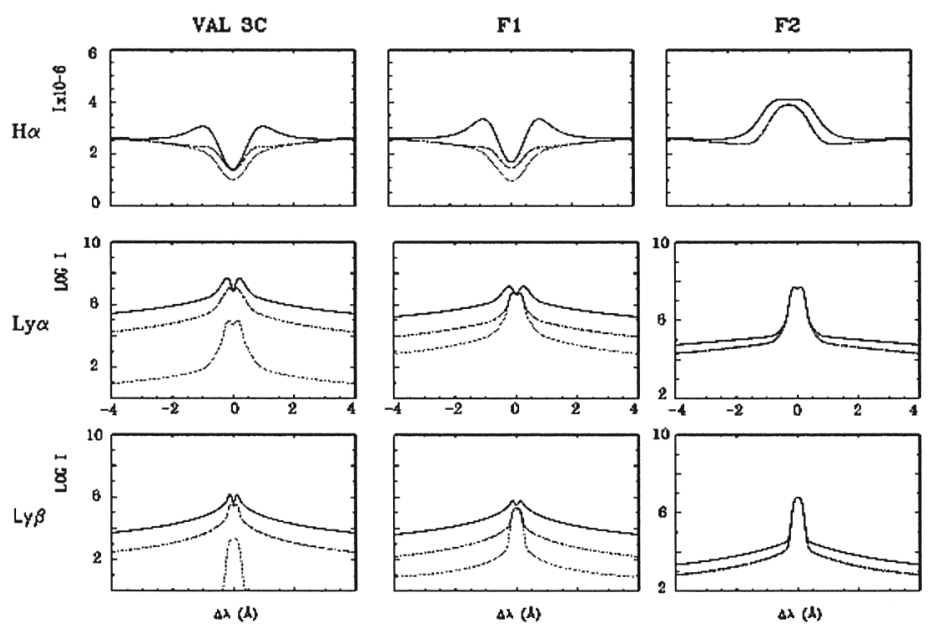

Figure 2. Line profiles for the different models bombarded by an electron beam (full lines) with energy flux $\mathcal{F}_{1}=510^{11} \mathrm{erg} \mathrm{cm}^{-2} \mathrm{~s}^{-1}$ and low energy cut-off $E_{1}=20 \mathrm{keV}$ and a proton beam (dotted-dashed lines) with energy flux $\mathcal{F}_{1}=510^{10} \mathrm{erg} \mathrm{cm}^{-2} \mathrm{~s}^{-1}$ and $E_{1}=150 \mathrm{keV}$. $\delta=4$ was adopted for all cases. 

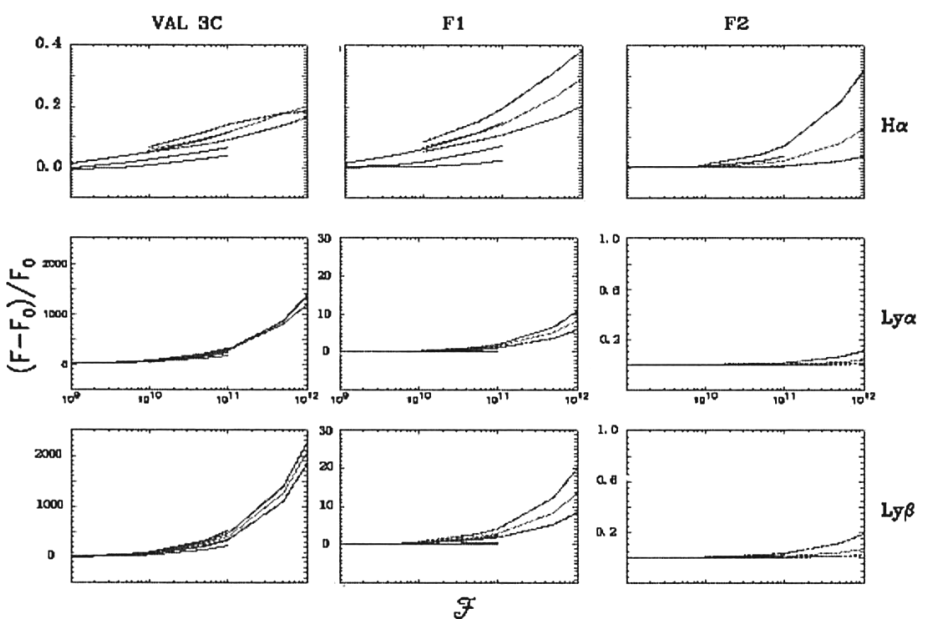

Figure 3. Dependence of the $\mathrm{H} \alpha$ line flux contrast on the power index $(3,4$, and 5 correspond to the upper, middle and lower curves, respectively) and the total energy flux of the electron beam (dashed lines) and the proton beam (solid lines).

the wings of the $\mathrm{H} \alpha$, Ly $\alpha$ and $\mathrm{Ly} \beta$ lines caused by electron beam bombardment is much larger than that by proton beam. Moreover, compared to the Fig. 1 in Paper I, the line intensities in Figure 1 are less stronger, and less sensitive to the values of $\delta$. This is consistent with the result given by Kasparova \& Heinzel (2002).

\section{A Method to Deduce the Particle Total Energy Input Flux}

Based on our results, we proposed a method to deduce the particle total energy input flux $\mathcal{F}_{1}$. We defined the $\mathrm{H} \alpha$ line flux contrast as (F-F0)/F0, where $\mathrm{F}$ and F0 are the flux of the non-thermal line profile and of the thermal line profile respectively. Figure 3 depicts the dependence of the $\mathrm{H} \alpha$ line flux contrast on the total energy flux and the power index of the electron beam (dashed lines) and the proton beam (solid lines). In fact, the observed flare impulsive $\mathrm{H} \alpha$ line profile is the non-thermal one, while the pre-flare line profile can be taken as the thermal one, assuming that the temperature structure changes not so much. Thus, the line flux contrast can be obtained from the observation. Using the theoretical curves in Fig.4, and knowing the particle power index by other methods (such as hard X-ray or radio emission), we can then deduce the particle total energy flux $\mathcal{F}_{1}$.

As an example, we used an $\mathrm{H} \alpha$ profile of the 2002 July $233 \mathrm{~B} / \mathrm{X} 4.8$ flare at 00:32:20 UT, and took $\delta=4$ from RHESSI data for the electron beam. Using the corresponding theoretical curve in Fig.3, we obtain the total energy flux of the electron beam being $110^{11} \mathrm{erg} \mathrm{cm}^{-2} \mathrm{~s}^{-1}$. By use of these parameters the observed $\mathrm{H} \alpha$ line profile can be well reproduced (Xu et al. 2003). 


\section{Conclusion}

1. In the case of electron bombardment, the intensity of $\mathrm{H} \alpha$ line wings will increase by a factor $1.5 \sim 2$, the line will be widely broadened and show strong central reversal. Ly $\alpha$ and Ly $\beta$ line wings will be enhanced more than $1 \sim 2$ orders of magnitude. The line profiles are good tools for diagnosing the total energy flux, but less sensitive to the power index.

2. In the case of proton bombardment, the enhancements of $\mathrm{H} \alpha$, $\operatorname{Ly} \alpha$ and $\operatorname{Ly} \beta$ lines are much less than that in the case of electron bombardment.

3. Based on $\mathrm{H} \alpha$ line flux measurement, we proposed a method to deduce the particle total energy flux $\mathcal{F}_{1}$.

Acknowledgments. This work is supported by two funds (No. 49990451 and No.10333040) from NSFC, and a key program (G20000784) from NKBRSF. M. D. Ding is also supported by TRAPOYT.

\section{References}

Aboudarham, J. \& Hénoux, J. -C. 1987, A\&A, 174, 270

Canfield, R. C. \& Chang, C. R. 1985, ApJ, 295, 275

Chambe, G. \& Hénoux, J. -C. 1979, A\&A, 80, 123

Emslie, A. G. 1978, ApJ, 224, 241

Fang, C., Hénoux, J. -C. \& Gan, W. Q. 1993, A\&A, 274, 917 (Paper I)

Fang, C., Feautrier, N. \& Hénoux, J. -C. 1995, A\&A, 297, 854

Hénoux, J. -C., Fang, C. \& Gan, W. Q. 1993, A\&A, 274, 923 (Paper II)

Kasparova, J. \& Heinzel, P. 2002, A\&A, 382, 688

Kazantsev, S. A. \& Hénoux, J. -C. 1995, Polarization Spectroscopy of Ionized Gases, Kluwer Acad. Publ., Dordrecht.

Machado, M. E., Avrett, E. H., Vernazza, J. E. et al. 1980, ApJ, 242, 336

Vernazza, J. E., Avrett, E. H. \& Loeser, R. 1981, ApJS, 45, 635

Xu, Z., Firstova, N. M. \& Fang, C. 2003, ChJAA, 3, 266

Zharkova, V. V. \& Kobylinskii, V. A. 1993. Sol. Phys., 143, 259 